\title{
Retrieval of aerosol optical thickness for desert conditions using MERIS observations during the SAMUM campaign
}

\author{
By TILMAN DINTER ${ }^{1 *}$, WOLFGANG VON HOYNINGEN-HUENE ${ }^{1}$, JOHN P. BURROW ${ }^{1}$, \\ ALEXANDER KOKHANOVSKY ${ }^{1}$, EIKE BIERW IRTH ${ }^{2}$, MANFRED WENDISCH ${ }^{2}$, DETLEF \\ MÜLLER ${ }^{3}$, RALPH KAHN ${ }^{4}$ and MOHAMMED DIOURI ${ }^{5}, \quad{ }^{1}$ Institute of Environmental Physics, \\ University of Bremen, Otto-Hahn-Allee 1, 28359 Bremen, Germany; ${ }^{2}$ Institute for Atmospheric Physics, Johannes \\ Gutenberg University, Johann-Joachim-Becher-Weg 21, 55099 Mainz, Germany; ${ }^{3}$ Leibniz Institute for Tropospheric \\ Research, Permoserstr. 15, 04318 Leipzig, Germany; ${ }^{4}$ Jet Propulsion Laboratory, California Institute of Technology \\ (Caltech), 4800 Oak Grove Drive, 91109 Pasadena, California, USA; ${ }^{5}$ Faculty of Sciences, University Mohammed I, \\ B.P. 524, Oujda 60000, Morocco
}

(Manuscript received 10 February 2008, in final form 14 August 2008)

\begin{abstract}
Approximately $30 \%$ of the land surface is arid, having desert or semi-desert conditions. Aerosol originating from these regions plays a significant role in climate and atmospheric chemistry of the atmosphere. Retrieving aerosol properties from space-borne platforms above desert conditions, where the surface reflectance is usually very bright, is a challenging task. The proportion of the surface to top of atmosphere (TOA) reflectance can reach values over $90 \%$, especially for wavelength above $500 \mathrm{~nm}$. For these reasons detailed knowledge of aerosol and surface optical properties from these regions is required to separate atmosphere from intrinsically bright surfaces.

An approach to retrieve aerosol properties over arid and semi-arid regions based on the Bremen Aerosol Retrieval (BAER) has been developed and validated within the Dust Aerosol Retrievals from Space-Born Instruments (DREAMS) Project, which is part of the Saharan Mineral Dust Experiment (SAMUM, 2006). Combining measurements of the backscattered radiation from the Medium Resolution Imaging Spectrometer (MERIS) instrument aboard Environmental Satellite (ENVISAT) and ground-based measurements in Morocco in radiation closure experiments yields the aerosol optical properties of mineral dust at selected locations.
\end{abstract}

\section{Introduction}

The recognition that anthropogenic activity directly and indirectly is modulating climate (IPCC, 2007), and the observation of increasing desertification, which, as a consequence, results in increasing amounts of atmospheric dust, has turned the attention of the scientific community on the importance of improving our understanding of aerosol. In this context airborne mineral dust plays an important role, having bio-geochemical impact on the ecosystem and influencing locally and globally atmospheric radiative forcing (Haywood and Bougher, 2000; Hsu et al., 2000; IPCC, 2007). As described in several studies (e.g. Kaufman et al., 2005), the dust from the Sahara is often transported out

\footnotetext{
* Corresponding author.

e-mail: dinter@iup.physik.uni-bremen.de

DOI: $10.1111 /$ j.1600-0889.2008.00391.x
}

of the boundary layer into free troposphere and then travels thousands of kilometres across the Atlantic or into northern Europe. For this reason, it is especially important to understand the processes controlling the formation, transport and fate of dust aerosols originating from Saharan source regions. Therefore, adequate satellite remote sensing observation techniques for mineral dust are required, which can be used over a wide variety of surface conditions, especially in source regions.

The Bremen aerosol retrieval algorithm (BAER) has been developed to retrieve aerosol optical properties above land and ocean surfaces. A detailed description of the BAER algorithm and its application for the retrieval of aerosol properties are discussed elsewhere (von Hoyningen-Huene et al., 2003). Predominantly existing retrieval approaches for aerosol optical thickness work for known low reflecting surface conditions. The bright surface of desert and semi-arid regions is the most challenging application for BAER. In desert regions, the spectral surface 
reflectance is very high in the red part of visible spectrum and near infrared, but decreases relatively fast in the blue for wavelengths shorter $500 \mathrm{~nm}$. This behaviour contrasts to that of, for example, clouds or snow, which are predominantly spectral neutral. To account for increased surface reflectance, associated with these very bright surfaces types, BAER needed to be modified. The radiation closures of the SAMUM experiment have been used to investigate aerosol (von Hoyningen-Huene et al., 2008) and surface (Bierwirth et al., 2008) properties and their interaction with the retrieval of aerosol optical thickness. A brief overview of the used satellite instrument and the usual aerosol retrieval techniques in the UV/VIS region will be given in Section 2. A description of the optimization of BAER for desert conditions will be discussed in Section 2.2 and validated in Section 4.

Examples of aerosol optical thickness derived by using the BAER algorithm over Sahara region during SAMUM campaign in South Morocco will be shown in this paper. The evaluations reveal various dust sources, which are important contributors to airborne dust transported over long distances. The aerosol optical thickness and surface reflectance are determined simultaneously in the algorithm using look-up tables to match the satellite observed spectral top of atmosphere (TOA) radiance. Reduced Resolution Level 1 data of the MEdium Resolution Imaging Spectrometer (MERIS), which is an radiometer on the Environmental Satellite (ENVISAT), are used. The instrument gives TOA radiance at 15 channels in the wavelength range of $400-1000 \mathrm{~nm}$.

\section{Satellite instrument and methodology}

Several algorithms for remote sensing of aerosol properties over land using different kind of sensors have already been developed. The BAER algorithm is generic and can be applied to any calibrated measurement of backscattered radiation, yielding the TOA spectral reflectance as a function of wavelength, especially those having sufficient channels in the short-wave region. In this context, it has been applied to the data from the sensors: Sea-Viewing Wide Field-of-View Sensor (SeaWiFS; http://oceancolor.gsfc.nasa.gov/SeaWiFS/), Moderate-Resolution Imaging Spectroradiometer (MODIS; http://modis.gsfc.nasa.gov/), MERIS (Rast et al., 1999; Bézy et al., 2000) and Scanning Imaging Absorption Spectrometer for Atmospheric Chartography (SCIAMACHY; Burrows et al., 1995; Bovensmann et al., 1999).

The standard MODIS aerosol retrieval algorithm over land uses the dark target approach (Kaufman et al., 1997a,b) and assumes a fixed relation between $0.47,0.64$ and the $2.1 \mu \mathrm{m}$ channels, respectively. This is a valid assumption for most vegetated land surfaces. But over desert regions these values deviates significantly from this assumption. For these conditions, there are substantial areas with data gaps in the standard MODIS aerosol product over land.
The BAER algorithm as well as the 'Deep Blue' algorithm from Hsu et al. (2004) uses the blue channels of satellite instruments for separation between surface and atmosphere. This is possible, because of the decreasing surface reflectance in this spectral regions. For dark surfaces (vegetation, 'brown' bare soil) this is an appropriate approach, whereas over desert and other bright reflecting surfaces this leads to an not negligible overestimation of the aerosol optical thickness. To avoid this problem Hsu et al. (2004) use as an a priori knowledge a surface reflectance determined from a clear-scene database for a given geolocation. Following this approach, this study is focusing on an improvement of the model for the spectral and bidirectional reflecting surface properties and its integration within the retrieval procedure. For this a surface reflectance data set for the SAMUM region based on atmospheric corrected MERIS data has been build up. Comparisons of this database with aircraft measurements are shown in Section 4.3.

\subsection{The Medium Resolution Imaging Spectrometer (MERIS)}

In this paper, retrievals of aerosol optical properties have been inverted from measurements made by the MERIS instrument, which flies on the European satellite ENVISAT.

Environmental Satellite was launched on the 1 March 2002. It flies in a sun synchronous orbit in descending mode, having an inclination of $82^{\circ}$ and a resulting equator crossing time of around 10 a.m.

The MERIS, medium resolution instrument, is a hyperspectral instrument. The backscatterd upwelling radiation from atmosphere and surface is directed into the instrument, which comprises a spectrometer and a 2-D array detector arrangement. Observations of the Earth shine and the extraterrestrial solar irradiance yield the spectral reflectance at the TOA for the particular viewing direction. The instrument has 15 spectral channels, which operate between 400 and $1000 \mathrm{~nm}$. The spatial resolution of one pixel is $1.04 \times 1.2 \mathrm{~km}$ reduced and $260 \times 300 \mathrm{~m}$ full resolved for all 15 channels. The swath width amounts $1150 \mathrm{~km}$ and yields to a global coverage every $3 \mathrm{~d}$. MERIS is a wellcalibrated instrument and is qualified for evaluations over bright reflecting surfaces, because there is no saturation problem with high radiation input in each of the spectral channels up to an albedo of 1.0 (Bézy et al., 2000). Furthermore it offers a successful mixture of spectral and spatial resolution and coverage.

\subsection{The BAER algorithm}

An overview of the steps within the extended BAER approach is presented in Fig. 1. The BAER method determines an aerosol reflectance by eq. (1) and has been extended for the purpose of retrievals over desert: 


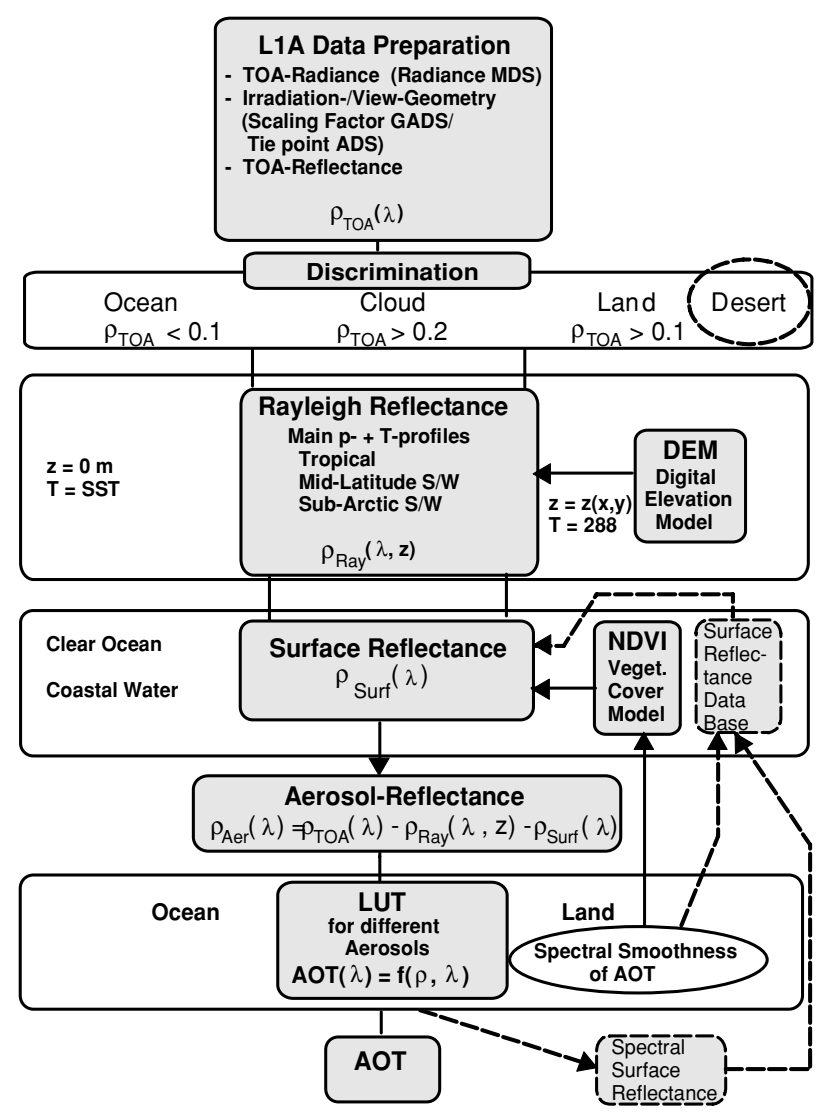

Fig. 1. Overview of the steps used in the framework of the retrieval method (BAER) for the derivation of the columnar aerosol optical thickness.

(1) Subtracting the path reflectance produced by the Rayleigh scattering for the geometry of illumination and observation. Correcting air masses by ground pressure using a digital elevation model for the surface elevation.

(2) Correcting the surface reflectance.

Over land: using a model of a linear mixture of reflectance spectra of vegetation and bare soil, tuned by Normalized Differential Vegetation Index (NDVI).

Over ocean: linear mixing of coastal water and clean ocean using Normalized Differential Phytoplankton Index (NDPI).

(3) A new land class 'Desert' has been implemented, using brighter bare soil spectra, if NDVI is close to zero.

(4) The use of constraints for the spectral aerosol optical thickness as a function of wavelength.

In this manner, BAER retrieves spectral AOT by separating the effect of aerosol on the backscattered radiation from those of surfaces. As a result the spectral surface reflectance is generated simultaneously for a given ground scene. The main problem is the selection and consideration of the variability of bare soil spectra.
To distinguish between cloudy and cloud free pixels from MERIS observations we use a three step approach. This based on the following three assumptions:

(1) First we use a simple threshold approach. That means if the TOA reflectance of channels 2, 3 and 4 (443, 490 and $510 \mathrm{~nm})$ exceeds a limit $\left(\rho_{\text {TOA }}>0.3\right)$ we assign this pixel as cloudy.

(2) If the ratio of the TOA reflectance for the first two channels $(412$ and $443 \mathrm{~nm})$ is lower than $\rho_{\text {TOA }}(412 \mathrm{~nm}) / \rho_{\text {TOA }}(443 \mathrm{~nm})<1.04$.

(3) The standard deviation of a centre pixel of a $5 \times 5$ pixel mask to the mean of this mask is a value for the gradient of this pixel to the neighbour pixels. This assign mainly the cloud borders and high reflectance variations within clouds. If the relation of the standard deviation to the mean of a $5 \times 5$ pixel mask is lager than $\sigma / \overline{\mathrm{AOT}(5 \times 5)}>0.005$ the pixel is masked as cloudy.

These values are working quite well for the region of SAMUM campaign. But improving this cloud parameters for independent retrievals on lager scales (beyond SAMUM region) is subject of further investigations.

The main equation (Kaufman et al., 1997a; von HoyningenHuene et al., 2003) used in this work can be formulated as follows:

$$
\begin{aligned}
& \rho_{\text {Aer }}\left(\lambda, z_{0}, z_{S}\right)=\rho_{\mathrm{TOA}}\left(\lambda, z_{0}, z_{S}\right) \\
& -\rho_{\text {Ray }}\left(\lambda, z_{0}, z_{S}, p_{\text {Surf }}(z)\right) \\
& -\frac{T\left(\lambda, M\left(z_{0}\right)\right) \cdot T\left(\lambda, M\left(z_{S}\right)\right) \cdot A_{\text {Surf }}(\lambda)}{1-A_{\text {Surf }}(\lambda) \cdot \rho_{\text {Hem }}(\lambda)},
\end{aligned}
$$

where $\rho_{\text {Aer }}$ is the aerosol and $\rho_{\text {TOA }}$ the TOA reflectance, calculated by the normalization of measured TOA radiance $L(\lambda)$ to the extraterrestrial irradiance $E_{0}(\lambda)$ multiplied by the airmass factor $M_{0}$ :

$\rho_{\mathrm{TOA}}(\lambda)=\frac{\pi L(\lambda)}{E_{0}(\lambda)} \cdot M_{0}$.

$\rho_{\text {Ray }}\left(\lambda, z_{0}, z_{S}, p_{\text {Surf }}(z)\right)$ is the path reflectance of the Rayleigh scattering whereas $p_{\text {Surf }}(z)$ is the surface pressure depending on the elevation of the ground for the corresponding pixel. The information of the elevation comes from the gtopo30 database (q. v. http://edc.usgs.gov/sitemap.html).

$T\left(\lambda, M\left(z_{0}\right)\right)$ and $T\left(\lambda, M\left(z_{S}\right)\right)$ are the total atmospheric transmission for the zenith distance of illumination $z_{0}$ and viewing geometry $z_{S}$ whereas $M$ is the air mass factor. The transmission factors containing direct and diffuse transmission for both radiation directions $z_{0, S} \cdot \rho_{\mathrm{Hem}}(\lambda)$ is the hemispheric atmospheric reflectance. The influence of the hemispheric reflectance over low reflecting surfaces (e.g. ocean or vegetation in the blue spectral region) is less important but not negligible over bright surfaces like desert. Total transmissions and hemispheric reflectance are determined by parametrization derived from radiative transfer calculations (Kokhanovsky et al., 2005; von Hoyningen-Huene 


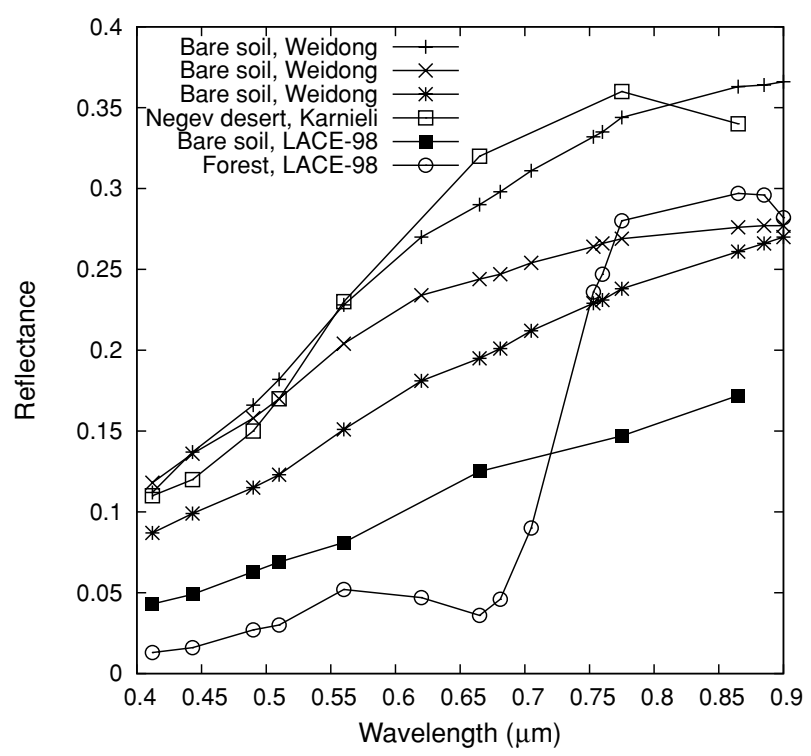

Fig. 2. Surface reflectance spectra of different bare soils, showing the variability of surface properties over the desert.

et al., 2007). The radiative transfer calculations are made using an Henyey-Greenstein asymmetry parameter of $g=0.7$. The transmissions and hemispheric reflectance are calculated for a AOT range of 0.0-2.5 and parametrized by polynomial coefficients.

The investigations with the SAMUM data have shown that consideration of the surface albedo $A_{\text {Surf }}(\lambda)$ as a Lambert ground is not sufficient. In this study it has been shown that a simple cosine law does not match the angle dependence of desert ground reflectance. Therefore, an improved surface bidirectional reflectance distribution function (BRDF) model is implemented in BAER and is described in Section 2.3.

Figure 2 shows several surface spectra, including data for bright bare soil conditions. Since the bare soil over the Sahara region is more or less the only surface influence, the variability of the soil type directly affects the spectral AOT. Therefore, a regional data set with an adequate surface spectra is required. This needs to be established iteratively by the BAER approach, starting with a priori assumptions for the region of interest. For the SAMUM region, the spectrum from Weidong et al. (2002) as $\rho_{\text {Soil }}(\lambda)$ and a vegetation spectrum $\rho_{\text {Veg }}(\lambda)$ from closure experiment Lindenberg Aerosol Characterization Experiment (LACE98) are used (see von Hoyningen-Huene et al., 2003).

\subsection{Improved surface model}

Using MERIS overflights, intercomparisons of retrieved aerosol optical thickness and ground-based sun photometer measurements have been made. These intercomparisons have shown systematic deviations depending on viewing geometry. Since the phasefunctions in von Hoyningen-Huene et al. (2008), see

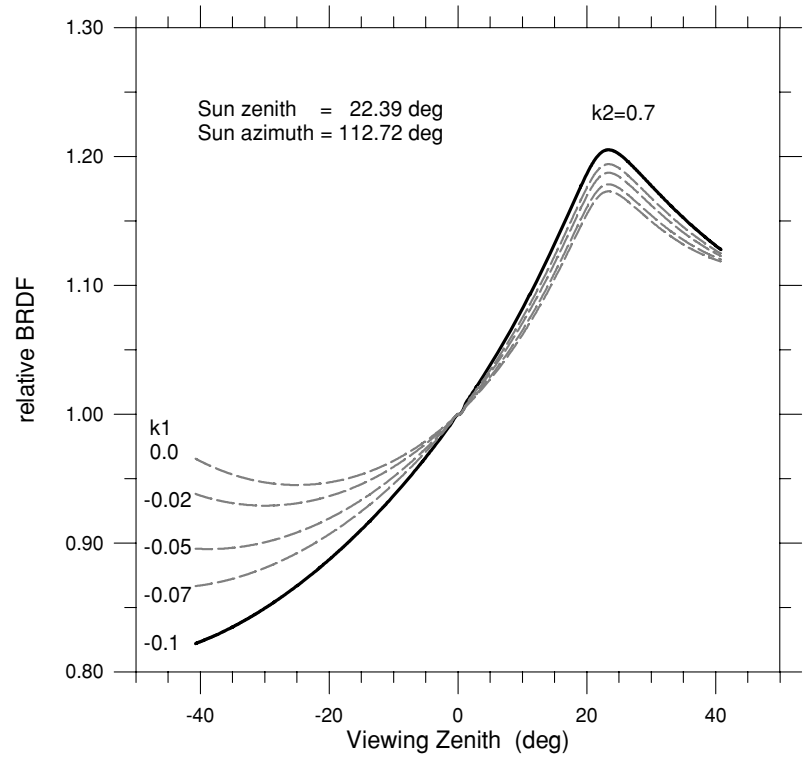

Fig. 3. Normalized BRDF depending on the viewing angle with fixed solar zenith and phase angle. $k_{1}$ determines the relation between forward and backscattering and $k_{2}$ is a parameter controlling the peak (the anisotropy) of the BRDF.

Fig. 4, do not support the observed deviations in viewing geometry, the observed effects need to be evoked by surface anisotropy. Therefore the surface reflectance model used by the BAER approach needs to be extended for anisotropy effects of surface.

The following surface reflectance model is used in this study:

$$
\begin{aligned}
\rho_{\mathrm{Surf}}(\lambda)= & \frac{F}{\mathrm{BRDF}^{*}} \cdot\left[C_{\mathrm{Veg}} \cdot \rho_{\mathrm{Veg}}(\lambda)\right. \\
& \left.+\left(1-C_{\mathrm{Veg}}\right) \cdot \rho_{\mathrm{Soil}}(\lambda)\right]
\end{aligned}
$$

with $F$ as scaling factor, adapting the spectral surface model to the conditions within the scene. This scaling is made for the wavelength $0.665 \mu \mathrm{m}$ (MERIS channel 7).

$F=\frac{\rho_{\mathrm{TOA}}(665 \mathrm{~nm})-\rho_{\mathrm{Ray}}(665 \mathrm{~nm})-\rho_{\mathrm{Aer}}(665 \mathrm{~nm})}{C_{\mathrm{Veg}} \cdot \rho_{\mathrm{Veg}}(665 \mathrm{~nm})+\left(1-C_{\mathrm{Veg}}\right) \cdot \rho_{\mathrm{Soil}}(665 \mathrm{~nm})}$.

$C_{\text {Veg }}$ is the vegetation fraction using an atmospheric corrected NDVI, calculated with channel $13(0.865 \mu \mathrm{m})$ and channel 7 $(0.665 \mu \mathrm{m})$ of MERIS and BRDF* is a normalized function considering the surface anisotropy relative to nadir viewing (see Fig. 3).

$\operatorname{BRDF}^{*}\left(z_{0}, z_{S}, \phi\right)=\frac{\operatorname{BRDF}\left(\mathrm{z}_{0}, \mathrm{z}_{\mathrm{S}}, \phi\right)}{\operatorname{BRDF}\left(\mathrm{z}_{0}, \mathrm{z}_{\mathrm{S}}=0, \phi\right)}$.

The original approach of von Hoyningen-Huene et al. (2006) does not consider the BRDF effects and $F$ was the only parameter for adapting the surface model. However, the radiation closure between ground-based results and satellite data within this study showed that significant deviations in AOT occur, if the surface anisotropy is neglected. To reduce deviations in AOT the surface 
anisotropy by the Rahman-Pinty-Verstraete BRDF model (Pinty et al., 1990; Verstraete et al., 1990; Rahman et al., 1993a,b; Maignan et al., 2004; Sinyuk et al., 2007) will be introduced as follows:

$\mathrm{BRDF}=k_{0} \cdot \frac{\cos ^{k_{2}-1} z_{0} \cdot \cos ^{k_{2}-1} z_{S}}{\left(\cos z_{0}+\cos z_{S}\right)^{1-k_{2}}} \cdot P(\theta) \cdot(1+R(\theta))$.

Thereby

$$
P(\theta)=\frac{1-k_{1}^{2}}{\left(1+k_{1}^{2}-2 \cdot k_{1} \cdot \cos (\pi-\theta)\right)^{3 / 2}}
$$

is a function of anisotropic scattering of the surface, where $\theta$ is the phase angle $\cos \theta=\cos z_{0} \cdot \cos z_{S}+\sin z_{0} \cdot \sin z_{S} \cdot \cos \phi$. $(1+R(\theta))$ describes the 'glint' region.

$R(\theta)=\frac{1-k_{0}}{1+g}$

$g=\left(\tan ^{2} z_{0}+\tan ^{2} z_{S}-2 \cdot \tan z_{0} \cdot \tan z_{S} \cdot \cos \phi\right)^{1 / 2}$.

The parameters $k_{0}, k_{1}$ and $k_{2}$ have to be determined to minimize the deviations of the retrieved AOT from satellite and ground.

$k_{0}$ is the parameter for the intensity of the surface reflectance and has less influence on the shape of the BRDF. As input of this parameter we use the result of $\left[C_{\mathrm{Veg}} \cdot \rho_{\mathrm{Veg}}(\lambda)+(1-\right.$ $\left.\left.C_{\mathrm{Veg}}\right) \cdot \rho_{\text {Soil }}(\lambda)\right]$ from eq. (3). For the conditions at the location of 'Porte au Sahara' $k_{2}=0.7$ and for $k_{1}=-0.1$ have been extracted as optimal parameters. The same parameters at the site Ouarzazate also lead to a significant reduction of deviations between retrieved satellite and ground-based AOT.

Assuming a 'black-sky' albedo, in other words neglecting the diffuse hemispherical part ('white-sky' albedo), the resulting surface reflectance $\rho_{\text {Surf }}(\lambda)$ can be treated as equivalent to the albedo $A_{\text {Surf }}(\lambda)$. The increase of the diffuse hemispherical reflectance along with increasing atmospheric aerosol loading is corrected by $\rho_{\mathrm{Hem}}(\lambda)$ in eq. (1).

The present status cannot give experience on the regional variability of these parameters, especially, if different surface types exist. These parameters are found for dry stone desert type in Southern Morocco. However, the continuation of the analysis of deviations between retrieved and ground-based AOT and the analysis of POLDER (Polarization and Directionality of the Earth's Reflectances) data will give also parameters for other surface types (Maignan et al., 2004).

\subsection{Calculations of look-up tables (LUTs) for desert conditions}

The LUTs describe the relationships between TOA reflectance, aerosol reflectance and the AOT, respectively, for the different channels of the MERIS instrument. These calculations have been carried out with radiative transfer models (RTM) to establish look-up tables for the retrieval. This is made by using the

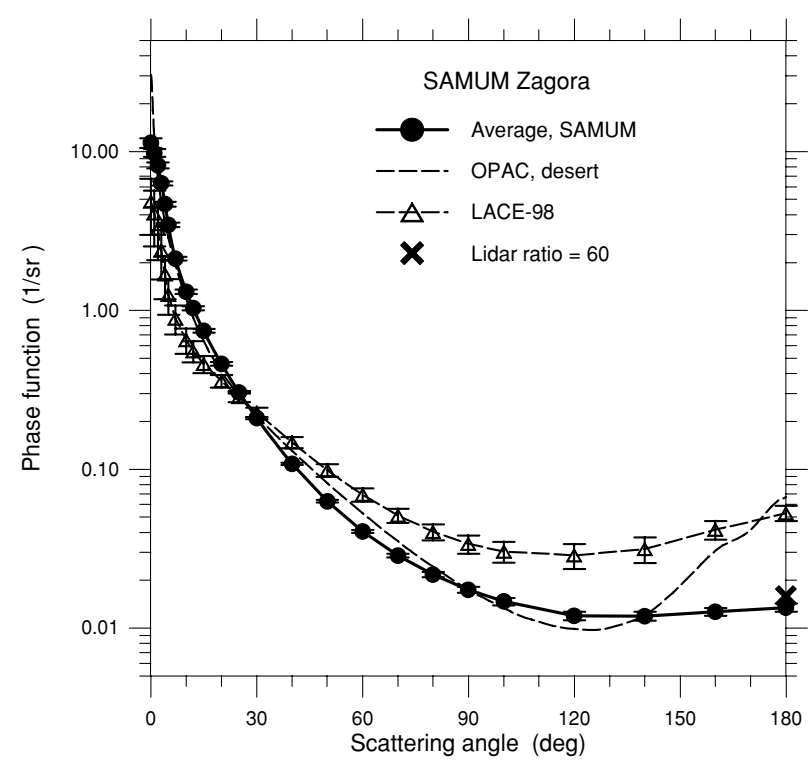

Fig. 4. Phasefunctions derived from CIMEL sky brightness measurements (von Hoyningen-Huene et al., 2008) and calculated by inversion model coupled inversion radiation transfer (CIRATRA; Wendisch et al., 1994; von Hoyningen-Huene and Posse, 1997, 1999). The model is based on the semi-empirical approach by Pollack and Cuzzi (1980). Value of $180^{\circ}$ is compared with derived value from lidar ratio (Heese et al., 2008; Tesche et al., 2008). OPAC is a widely used standard aerosol and cloud model using MIE theory (see Hess et al., 1998). LACE-98 is an example of a typical aerosol type over Germany (see also von Hoyningen-Huene et al., 2003).

radiative transfer code of Nakajima et al. (1988) for the solar illumination and satellite observation geometry within the scene. Similar results have been obtained using the RTM SCIATRAN (Rozanov et al., 2002). Aerosol reflectance is strongly dependent on illumination and viewing geometry. Since the RTM include multiple scattering, the effects of multiple scattering are automatically considered in the LUT.

The setup of LUT requires input parameters for the RTM. These are the aerosol optical thickness, the phasefunction and the single scattering albedo of the aerosol and the spectral surface reflectance. The aerosol optical thickness, the phasefunction and the single scattering albedo were determined using groundbased measurements (von Hoyningen-Huene et al., 2008) and the spectral surface albedo by aircraft measurements (Bierwirth et al., 2008).

Examples of calculated phasefunctions are shown in Fig. 4. This phasefunctions are calculated from ground-based CIMEL sky brightness measurements by using an inversion model based on a semi-empirical approach by Pollack and Cuzzi (1980) and was done in von Hoyningen-Huene et al. (2008). The curve shape shows a very smooth behaviour in the backward scattering region and no back scattering peak which is an outcome of nonspherical particles. Further information are available in the work of von Hoyningen-Huene et al. (2008). 


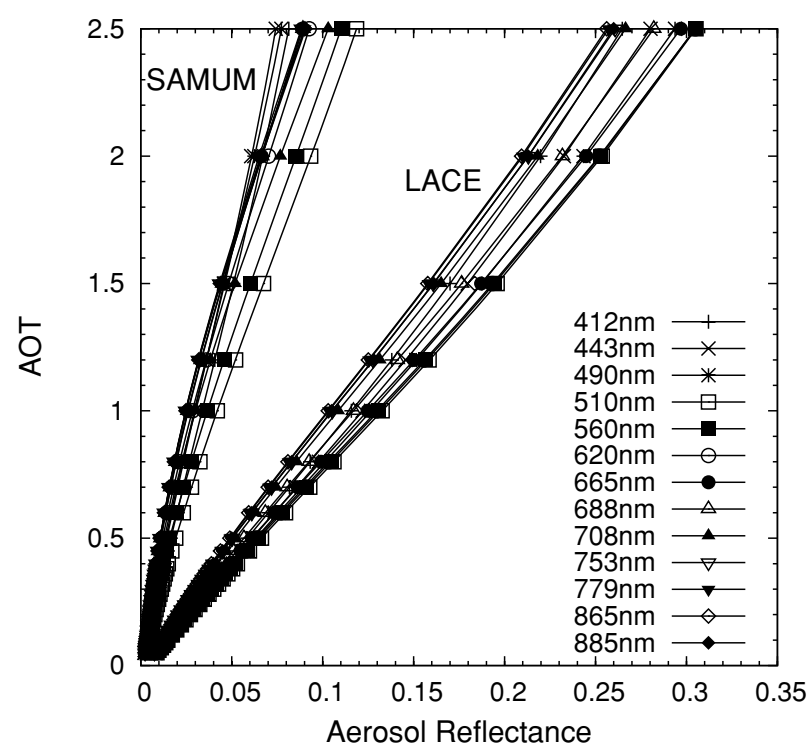

Fig. 5. Relation between aerosol optical thickness and aerosol reflectance, obtained by radiative transfer calculations by Nakajima et al. (1988) for the wavelengths of 13 MERIS channels. This plot shows the strong increasing of AOT for desert regions (SAMUM) with increasing aerosol reflectance compared to the LUT derived from measurements during LACE-98 campaign in Europe.

The results of the radiative transfer calculations are shown in Fig. 5. The LUT was calculated up to an aerosol optical thickness of 2.5. The AOT increases strongly with increasing aerosol reflectance, this means low deviation of the retrieved aerosol reflectance leads to a high variation in the AOT.

If we look to the variability of the anisotropy effects of the surface (see Section 2.3) we get differences of $\pm 20 \%$ in the considered surface albedo (see Fig. 3). This makes a difference for lower wavelength, where the surface reflectance is about 0.1 (see Fig. 2), of \pm 0.32 in the retrieved AOT. For larger wavelength $(>500 \mathrm{~nm})$, where the reflectance of bright surfaces may be greater than 0.3 (see Fig. 2), deviation of $\pm 20 \%$ leads to an over- or underestimation of the AOT of 1.15.

Therefore, we retrieve the AOT only for the first seven channels of MERIS (412-665 nm). The data for lager wavelength are extrapolated with the calculated Ångström coefficient to the six channels above $665 \mathrm{~nm}(682-885 \mathrm{~nm})$ to recalculate the atmospheric corrected surface reflectance.

Radiative transfer calculations have also shown that a brighter surface reflectance yields to an increase of the multiple scattering fraction as part of the aerosol reflectance. Thus, the surface has an important influence on the retrieved AOT. Other than for relative dark surface conditions, systematic LUTs for different surface conditions are required.

\section{Retrievals over desert regions}

The extended BAER approach has been tested over the Sahara region during the SAMUM experiment in Ouarzazate and Zagora
("Port au Sahara"), South Morocco (SAMUM, 2006). During the SAMUM experiment (12 May-7 June 2006) the following closure measurements have been made:

(1) Ground-based measurement for spectral AOT and skybrightness with a CIMEL 318 sun photometer to derive validation data and required aerosol phasefunctions for the desert dust.

(2) Spectral albedo measurements from aircraft (Bierwirth et al., 2008) to compare the retrieved spectral surface reflectance by BAER.

(3) Top of atmosphere radiance by the MERIS instrument for the retrieval of AOT and surface reflectance by BAER and for further closure studies to derive additional aerosol and surface parameters.

Inter-comparisons with the ground- and satellite-based data will be given in Section 4. First retrievals over desert region with the modified BAER are presented in this paper. Using the desert starting conditions described in Sections 2.2, 2.3 and 2.4, first retrievals of AOT and surface reflectance over Morocco and surrounding region have been obtained. As one example, Fig. 6 gives the regional pattern of AOT for MERIS channel 1 (412 nm) over land and ocean for the MERIS scene of 19 May 2006. The corresponding RGB image is given in Fig. 7.

\section{Validation of satellite aerosol retrieval}

\subsection{Comparison with ground-based measurements}

A validation of AOT for all MERIS overflights during the SAMUM campaign (12 May-7 June 2006) in Morocco is given in Fig. 8. The two CIMEL sun photometers for ground-based AOT measurements were placed at the airport of Ouarzazate as well as $36 \mathrm{~km}$ southeast of Zagora at 'Porte au Sahara'. The sun photometer in Ouarzazate was part of the AERONET network and the sun photometer in Zagora was contributed by the Institute of Environmental Physics, University of Bremen. For further information see corresponding publications (von Hoyningen-Huene et al., 2008).

First validations have been done by wavelength $440 \mathrm{~nm}$ of the sun photometer and $443 \mathrm{~nm}$ of the MERIS instrument, respectively. The retrieved AOT from satellite data is averaged over a $5 \times 5$ pixel mask of the corresponding geolocation. The comparisons show qualitative good agreements between retrieved AOTs with a correlation coefficient of 0.84 (see Fig. 8). The time of measurements and satellite overpass time are synchronized and have maximum deviation of $\pm 20 \mathrm{~min}$. Nonetheless strong temporal dynamics of aerosol loading in these regions could be responsible for deviations of ground- and satellite-based measurements because of differences in measuring time.

22 and 25 May 2006 show very high AOTs around 1.2 for ground measurements. The BAER algorithm detects these 
Fig. 6. Aerosol optical thickness at $443 \mathrm{~nm}$ (channel 2) retrieved from MERIS on 19 May 2006 showing Southern Morocco with aerosols source regions over the Sahara and high aerosol loading to the west direction. For the land class 'Desert' one surface spectrum with the highest spectral slope is assessed. The AOT values are gridded with a resolution of 0.05 degree.

Fig. 7. RGB composition of MERIS

channels 1-7 for the same scene as in Fig. 9 with added locations of SAMUM

experiment. The two sun photometers were located at Ouarzazate Airport ( $1100 \mathrm{~m}$ above sea level) and $36 \mathrm{~km}$ southeast of Zagora at 'Porte au Sahara' (680 m above sea level).
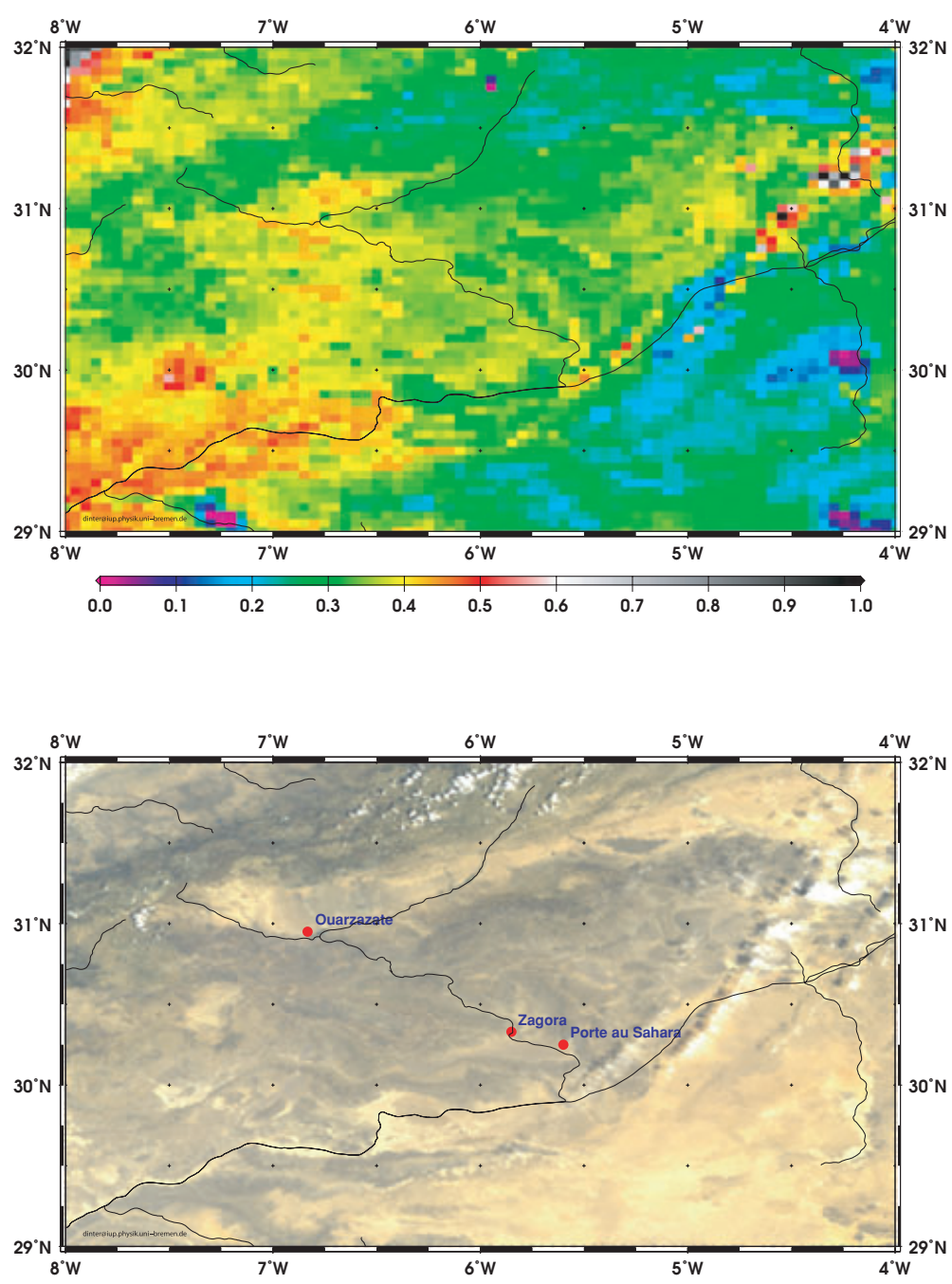

conditions as cloudy and disregards the relevant pixels from standard AOT retrieval.

In Fig. 9, comparisons of spectral AOT retrieved from satellite (MERIS BAER) and CIMEL ground-based instrument are shown. The AOTs of 13 MERIS channels are averaged over $5 \times 5$ pixels for the geolocation of the SAMUM AERONET site in Ouarzazate. The error-bars are the standard deviation for the $5 \times 5$ pixel mask. The sun photometer spectra in Fig. 9 are measured for the same time as the MERIS overpass time with maximum deviations of $\pm 20 \mathrm{~min}$. The MERIS AOT shows a higher Ångström coefficient than the sun photometer. For the first four MERIS channels the comparison shows good agreement but for wavelengths above $500 \mathrm{~nm}$ the deviation increases. This behaviour is due to the increasing of total TOA reflectance in relation to the aerosol reflectance, which is a consequence of increasing surface reflectance. So little deviations in the estimate of surface reflectance have a strong impact on the retrieved AOT.

\subsection{Comparison with MISR}

The Multiangle Imaging Spectroradiometer (MISR) flies aboard the TERRA satellite in a sun synchronous orbit and measures upwelling solar radiation scattered by Earth surface and atmosphere. The instrument measures for each pixel in a swath of $400 \mathrm{~km}$ width four short-wave spectral channels (446, 558, 672 and $866 \mathrm{~nm}$ ) at nine different viewing angles (forward and backward $70.5^{\circ}, 60.0^{\circ}, 45.6^{\circ}, 26.1^{\circ}$ and nadir view). With this feature the MISR aerosol retrieval algorithms are able to distinguish contributions in TOA reflectance from surface and atmosphere (Martonchik et al., 1998, 2002, 2004; Kahn et al., 2005). The MISR standard aerosol product reports AOT and aerosol type at $17.6 \mathrm{~km}$ spatial resolution. With the swath width of $400 \mathrm{~km}$ the MISR instrument has a poorer coverage than the MERIS instrument and get a global coverage every $9 \mathrm{~d}$. During SAMUM field campaign there were three dates (19, 28 May and 04 June 2006), where the MISR overpass covers the region of interest 


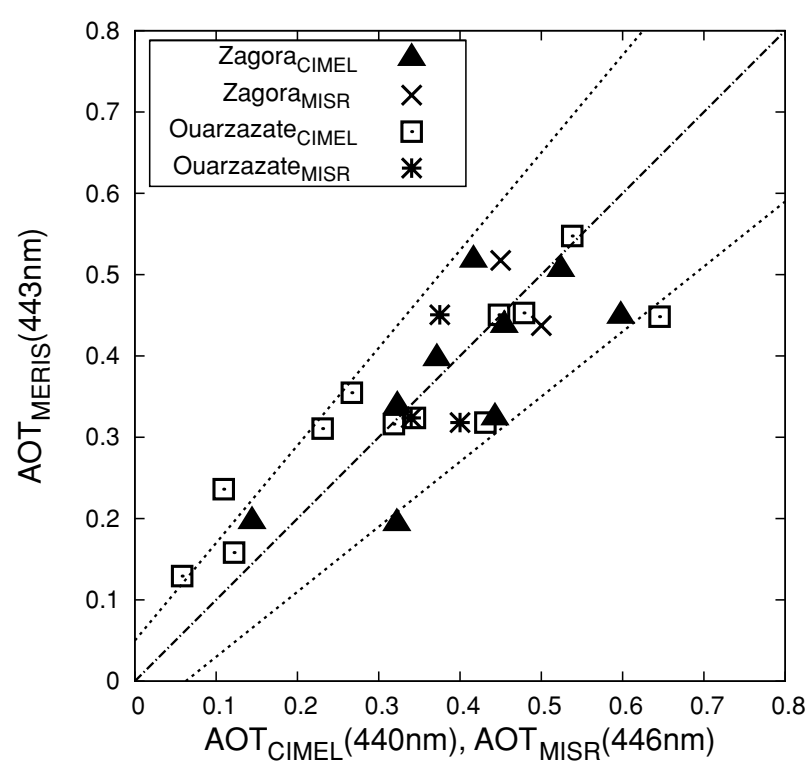

Fig. 8. AOT from CIMEL ground-based measurements and MISR satellite product versus MERIS AOT retrievals during SAMUM campaign(12 May-7 June 2006). The MERIS AOT is averaged over a $5 \times 5$ pixel mask of the corresponding geolocation. The chain dotted line is the one-to-one line and the dotted lines are the expected error of the MERIS BAER retrieval $( \pm 0.05 \pm 0.2 \cdot$ AOT $)$. The correlation coefficient is 0.84 .

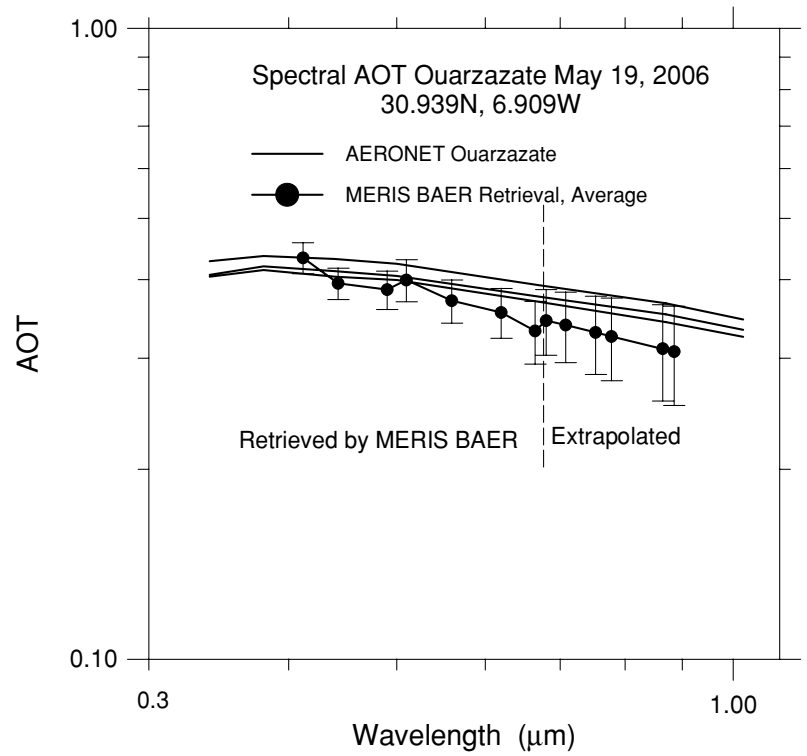

Fig. 9. Comparison of spectral AOT from CIMEL ground-based measurements and MERIS AOT retrievals over Ouarzazate for MERIS overflight 19 May 2006 10:51 UTC. Good agreement in the short wavelength region, whereas higher deviations in the wavelength region above $550 \mathrm{~nm}$ lead to an overestimation of the Ångström parameter.

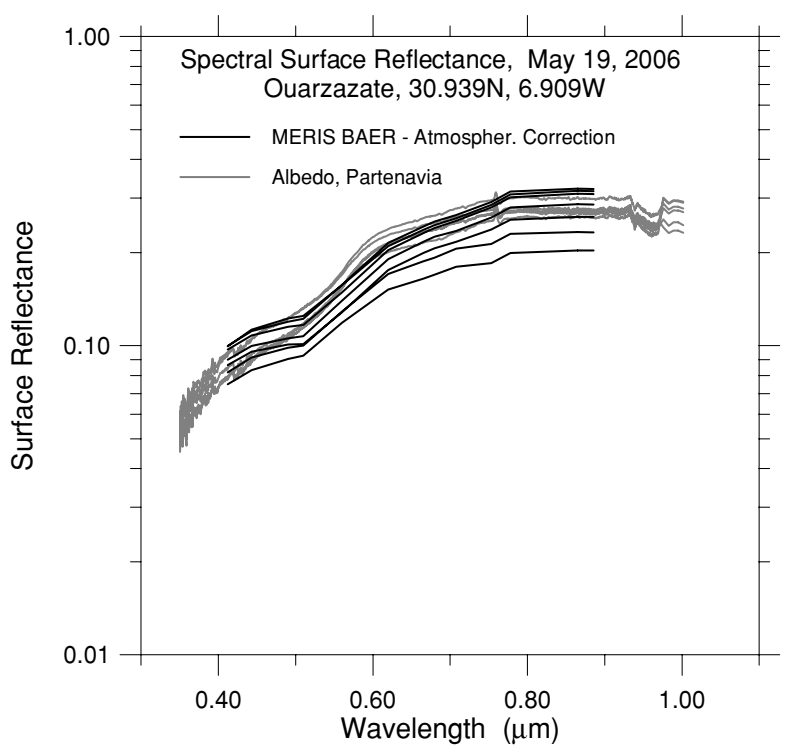

Fig. 10. Comparison of retrieved (by BAER) spectral surface reflectance with albedo measurements of aircraft flight project during SAMUM campaign, for the MERIS overpass 19 May 2006 10:51 UTC. The grey curves are measured by aircraft (Partenavia) near the airport $\left(30.939^{\circ} \mathrm{N}, 6.909^{\circ} \mathrm{W}\right)$ in Ouarzazate for the same time as MERIS overpass. The black curves are the retrieved surface reflectance of the collocated (to the position of aircraft) MERIS pixel.

(see Kahn et al., 2008). The comparisons between MISR and MERIS show good agreement with little deviation (see Fig. 8) which could be caused by different overpass times ( $\sim 30 \mathrm{~min})$.

\subsection{Comparison with measurements from aircraft}

If the BAER algorithm completes the AOT retrieval successfully, which means with a RMSD lower than 0.05 , the standard run is followed by an additional loop: The recalculation of surface albedo by inversion of eq. (1) with all retrieved parameters.

In Fig. 10, comparisons of surface reflectance from satellite and aircraft in the region of Ouarzazate for the 19 May 2006 are shown. For each aircraft measurement the next collocated pixel of MERIS measurement is evaluated. The TOA reflectance has been corrected by the atmosphere reflectance, in other words by the Rayleigh reflectance $\rho_{\text {Ray }}\left(\lambda, z_{0}, z_{S}, p_{\text {Surf }}(z)\right)$ and aerosol reflectance $\rho_{\text {Aer }}(\lambda)$.

First retrievals of recalculated surface albedo for the 19 May 2006 in the region of Ouarzazate show qualitative good agreement with air plane measurements from Bierwirth et al. (2008). The BAER algorithm reproduces the shape of the spectral dependence of the albedo in a correct way, but the deviation of the absolute adaptation in wavelength above $550 \mathrm{~nm}$ leads to a larger error in the retrieved aerosol optical thickness (see also Fig. 9). 


\section{Summary and conclusion}

First retrievals of aerosol optical thickness over arid and semiarid Saharan desert regions of the MERIS instrument aboard ENVISAT are obtained. These results have been analysed and validated in comparison with the ground-based observations.

It has been shown that the assumption of a Lambert ground leaded to unresolved surface effects in the AOT retrieval. Especially dependencies of the AOT results on viewing geometry were observed. Exclusion of the TOA reflectance anisotropy due to aerosol and molecular scattering, leads to the conclusion that further surface directional effects have to be taken into account.

Therefore, following improvements and modifications in the original BAER retrieval have been required to improve AOT results over brighter surfaces.

(1) The Rayleigh path reflectance $\rho_{\text {Ray }}\left(\lambda, z_{0}, z_{S}, p_{\text {Surf }}(z)\right)$ needs to be calculated considering the approximate value surface reflectance. The assumption of 'black' surface leads to biased spectral behaviour of AOT.

(2) The surface cannot be treated as a Lambert ground. The retrieval results suffered from surface anisotropy, depending on illumination and viewing geometry. Since the MERIS observations during SAMUM include the 'glint' region, clear indications for non Lambertian surface effects have been found.

(3) For the compensation of surface anisotropy the parameters of a BRDF model (Maignan et al., 2004; Pinty et al., 1990; Verstraete et al., 1990; Rahman et al., 1993a,b; Sinyuk et al., 2007) have been determined to minimize the deviations between retrieved AOT and ground-based observations. Thus a normalized BRDF model has been obtained, which is implemented into the surface model of BAER.

With these changes in the original BAER approach comparable AOTs with ground-based observations have been obtained. The results are now comparable within a $25 \%$ range in magnitude and in spectral slope (see Figs. 8 and 9). The correlation of retrieved AOT of $443 \mathrm{~nm}$ from ground- and satellite-based measurements improves from 0.22 to 0.84 .

First retrievals of recalculated surface albedo for the 19 May 2006 over Ouarzazate show qualitative good agreement with airplane measurements from Bierwirth et al. (2008) (see Fig. 10). The algorithm reproduces the spectral shape of the albedo in a correct way, but the deviation of the absolute adaption for wavelengths above $550 \mathrm{~nm}$ leads to a larger error in the retrieved aerosol optical thickness.

This is the first time a retrieval is presented to analyse spectral aerosol properties over bright reflecting desert regions for the MERIS instrument. To solve such challenging problem closure experiments like SAMUM are essential to get detailed knowledge about local atmosphere aerosol conditions. With that knowledge we were able to improve the BAER algorithm to those conditions. The key issue to retrieve aerosol optical thickness from TOA reflectance is the separation between atmosphere and surface reflectance. Because of high variability and no atmospheric independent characteristic, like the NDVI, we need a priori knowledge about the surface reflectance. This knowledge comes from experimental measurements like the SAMUM campaign. To retrieve larger regions from satellite, not everywhere measurements are available, we need to use a database of surface reflectivity using, for example, the minimum reflectivity technique (Herman et al., 1997; Koelemeijer et al., 2003).

The radiative transfer calculations for the determination of LUT showed that brighter surface reflectance leads to an increased contribution of multiple scattering. Therefore, LUT calculated for dark (or even 'black') surface conditions will underestimate higher AOT.

\section{Acknowledgments}

We thank ESA for providing us with the MERIS Level 1 data. Funding for this work came from the German institution DFG (Deutsche Forschungsgemeinschaft). We acknowledge exchange of data, measurements and information within the SAMUM group of researchers (SAMUM, 2006).

\section{References}

Bézy, J. L., Delwart, S. and Rast, M. 2000. MERIS—a new generation of ocean-color sensor onboard ENVISAT. ESA Bull. 103, 48-56.

Bierwirth, E., Wendisch, M., Ehrlich, A., Heese, B., Otto, S. and coauthors. 2008. Spectral surface albedo over Morocco and its impact on the radiative forcing of Saharan dust. Tellus 61B, doi:10.1111/j.16000889.2008.00395.x.

Bovensmann, H., Burrows, J. P., Buchwitz, M., Frerick, J., Noël, S. and co-authors. 1999. SCIAMACHY: mission objectives and measurement modes. J. Atmos. Sci. 56, 127-150.

Burrows, J. P., Hölzle, E., Goede, A. P. H., Visser, H. and Fricke, W. 1995. SCIAMACHY - Scanning Imaging Absorption Spectrometer for atmospheric chartography. Acta Astronaut. 35, 445-451.

Haywood, J. and Bougher, O. 2000. Estimates of the direct and indirect radiative forcing due to tropospheric aerosols: a review. Rev. Geophys. 38, 513-543.

Heese, B., Althausen, D., Dinter, T., Esselborn, M., Müller, T., Tesche, M. and Wiegner, M. 2008. Vertically resolved Dust Optical Properties during SAMUM: Tinfou compared to Ouarzazate. Tellus 61B, doi:10.1111/j.1600-0889.2008.00404.x.

Herman, J. R. and Celarier, E. A. 1997. Earth surface reflectivity climatology at 340-380 nm from TOMS data. J. Geophys. Res. 102, $28003-28011$.

Hess, M., Koepke, P. and Schult, I. 1998. Optical properties of aerosols and clouds: the software package OPAC. Bull. Am. Meteorol. Soc. 79, 831-844.

von Hoyningen-Huene, W. and Posse, P. 1997. Non-sphericity of aerosol particles and their contribution to radiative forcing. J. Quant. Spectrosc. Rad. Trans. 57, 651-668.

von Hoyningen-Huene, W., Wenzel, K. and Schienbein, S. 1999. Radiative properties of desert dust and its effect on radiative balance. $J$. Aerosol Sci. 30, 489-502. 
von Hoyningen-Huene, W., Freitag, M. and Burrows, J. P. 2003. Retrieval of aerosol optical thickness over land surfaces from top-of-atmosphere radiance. J. Geophys. Res. 108, 4260.

von Hoyningen-Huene, W., Kokhanovsky, A., Bruniquel-Pinel, V., Regner, P. and Barét, F. 2006. Simultaneous determination of aerosoland surface characteristics from top-of-atmosphere reflectance using MERIS on board of ENVISAT. Adv. Space Res. 37, 2172-2177.

von Hoyningen-Huene, W., Kokhanovsky, A. A., Wuttke, M., Buchwitz, M., Noël, S. and co-authors. 2007. Validation of SCIAMACHY topof-atmosphere reflectance for aerosol remote sensing using MERIS L1 data. Atmos. Chem. Phys. 7, 97-106.

von Hoyningen-Huene, W., Dinter, T., Kokhanovsky, A. A., Burrows, J. P. and Diouri, M. 2008. Radiative properties of desert dust for satellite remote sensing of aerosol optical thickness. Tellus 61B, doi:10.1111/j.1600-0889.2008.00405.x.

Hsu, N. C., Herman, J. R. and Weaver, C. 2000. Determination of radiative forcing of Saharan dust using combined TOMS and ERBE data. J. Geophys. Res. 105, 20 649-20661.

Hsu, N. C., Tsay, S., King, M. D. and Herman, J. R. 2004. Aerosol properties over bright-reflecting source regions. IEEE Trans. GRS $\mathbf{4 2}$, $557-569$.

Hsu, N. C., Tsay, S., King, M. D. and Herman, J. R. 2006. Deep blue retrievals of Asian aerosol properties during ACE-Asia. IEEE Trans. GRS 44, 3180-3195.

IPCC, B. 2007. Climate change. The physical science basis. contribution of working group 1 to the fourth assessment report of the intergovernmental panel on climate change (IPCC). In: Climate Change 2007 (eds. A. S. Solomon, D. Qin, M. Manning, M. Marquis, K. Averyt, and co-editors). Cambridge University Press, Cambridge.

Kahn, R. A., Gaitley, B. J., Martonchik, J. V., Diner, D. J. and Crean, K. A., 2005. Multiangle Imaging Spectroradiometer (MISR) global aerosol optical depth validation bases on 2 years of coincident Aerosol Robotic Network (AERONET) observations. J. Geophys. Res. 110, doi:10.1029/2004JD004706.

Kahn, R., Petzold, A., Wendisch, M., Bierwirth, E., Esselborn, M. and co-authors. 2008. Desert dust aerosol air mass mapping in the western Sahara, using particle properties derived from space-based multiangle imaging. Tellus 61B, doi:10.1111/j.1600-0889.2008.00398.x.

Kaufman, Y. J., Tanre, D., Remer, L. A., Vermote, E. F., Chu, A. and coauthors. 1997a. Operational remote sensing of tropospheric aerosol over land from EOS moderate resolution imaging spectroradiometer. J. Geophys. Res. 102, 51-67.

Kaufman, Y. J., Wald, A. E., Remer, L. A., Gao, B., Rong-Rong, L. and co-authors. 1997b. The MODIS 2.1- $\mu \mathrm{m}$ channel—correlation with visible reflectance for use in remote sensing of aerosol. IEEE Trans. GRS 35, 1286-1298.

Kaufman, Y. J., Koren, I., Remer, L. A., Tanre, D., Ginoux, P. and coauthors. 2005. Dust transport and deposition observed from the TerraModerate Resolution Imaging Spectroradiometer (MODIS) spacecraft over the Atlantic Ocean. J. Geophys. Res. 110, 1-16.

Koelemeijer, R. B. A., de Haan, J. F. and Stammes, P. 2003. A database of spectral surface reflectivity in the range $335-772 \mathrm{~nm}$ derived from 5.5 years of GOME observations. J. Geophys. Res. 108, 4070-4083.

Kokhanovsky, A. A., Mayer, B. and Rozanov, V. V. 2005. A parameterization of the diffuse transmittance and reflectance for aerosol remote sensing problems. Atmos. Res. 73, 37-43.

Maignan, F., Bréon, F.-M. and Lacaze, R. 2004. Bidirectional reflectance of Earth targets: Evaluation of analytical models using a large set of spaceborne measurements with emphasis on the Hot Spot. Remote Sens. Environ. 90, 210-220.

Martonchik, J. V., Diner, D. J., Kahn, R. A., Verstraete, M., Pinty, B. and co-authors. 1998. Techniques for the retrieval of aerosol properties over land and ocean using multiangle imaging. IEEE Trans. Geosci. Remote Sens. 36, 1212-1227.

Martonchik, J. V., Diner, D. J., Crean, K. and Bull, M. 2002. Regional aerosol retrieval results from MISR. IEEE Trans. Geosci. Remote Sens. 40, 1520-1531.

Martonchik, J. V., Diner, D. J., Kahn, R. A., Gaitley, B. J. and Holben, B. N. 2004. Comparison of MISR and AERONET aerosol optical depths over desert sites. J. Geophys. Res. Lett. 31, doi: 10.1029/2004GL019807.

Nakajima, T. and Tanaka, M. 1988. Algorithms for radiative intensity calculations in moderately thick atmospheres using a truncation approximation. J. Quant. Spectrosc. Radiat. Transfer 40, 51-69.

Pinty, B., Verstraete, M. M. and Dickinson, R. E. 1990. A physical model of the bidirectional reflectance of vegetation Canopies 2. Inversion and validation. J. Geophys. Res. 95, 11767-11775.

Pollack, J. B. and Cuzzi, J. N. 1980. Scattering by nonspherical particles of size comparable to a wavelength: a new semi-empirical theory and its application to tropospheric aerosols. J. Atmos. Sci. 37, 868-881.

Rahman, H., Verstraete, M. M. and Pinty, B. 1993a. Coupled surfaceatmosphere reflectance (CSAR) model 1. Model description and inversion on synthetic data. J. Geophys. Res. 98, 20779-20789.

Rahman, H., Pinty, B. and Verstraete, M. M. 1993b. Coupled surfaceatmosphere reflectance (CSAR) model 2. Semiempirical surface model usable with NOAA advanced very high resolution radiometer data. J. Geophys. Res. 98, 20 791-20801.

Rast, M., Bézy, J.-L. and Bruzzi, S. 1999. The ESA Medium Resolution Imaging Spectrometer MERIS-a review of the instrument and its mission. J. Remote Sens. 20, 1682-1701.

Rozanov, V. V., Buchwitz, M., Eichmann, K.-U., de Beek, R. and Burrows, J. P. 2002. SCIATRAN—a new radiative transfer model for geophysical applications in the $240-2400 \mathrm{~nm}$ spectral region: the pseudo-spherical version. Adv. Space Res. 29, 1831-1835.

SAMUM 2006. SAharan Mineral dUst experiMent. available at: http://www.tropos.de/samum/.

Sinyuk, A., Dubovik, O., Holben, B., Eck, T. F., Bréon, F.-M. and coauthors. 2007. Simultaneous retrieval of aerosol and surface properties from a combination of AERONET and satellite data. Remote Sensi. Environ. 107, 90-108.

Tesche, M., Ansmann, A., Müller, D., Althausen, D., Mattis, I. and coauthors. 2008. Vertical profiling of Saharan dust with Raman lidars and airborne HSRL in southern Morocco during SAMUM. Tellus 61B, doi:10.1111/j.1600-0889.2008.00390.x.

Verstraete, M. M., Pinty, B. and Dickinson, R. E. 1990. A physical model of the bidirectional reflectance of vegetation Canopies 1 . Theory. $J$. Geophys. Res. 95, 11 755-11765.

Weidong, L., Barét, F., Xingfa, G., Qingxi, T., Lanfen, Z. and co-authors. 2002. Relating soil surface moisture to reflectance. Remote Sens. Environ. 107, 90-108.

Wendisch, M. and von Hoyningen-Huene, W. 1994. Possibility of refractive index determination of atmospheric aerosol particles by ground based solar extinction and scattering measurements. Atmos. Environ. 28, 785-795. 